\title{
A novel technique for semiselective in situ bronchial artery perfusion in human lung retrieval
}

\author{
Bernhard Gohrbandt, MD, Gregor Warnecke, MD, Stefan Fischer, MD, MSc, Axel Haverich, MD, and \\ Martin Strueber, MD, Hannover, Germany
}

$\mathrm{T}$ he ideal strategy for pulmonary graft preservation remains elusive. Bronchial complications continue to occur after lung transplantation despite advances in operative technique and organ preservation. Airway ischemia of the graft may lead to tissue necrosis, resulting in bronchomalacia, formation of granulation tissue with subsequent strictures, or bronchial dehiscence followed by life-threatening subsequent vascular erosion. ${ }^{1,2}$ Anatomically, a dual blood supply to the lung consisting of the pulmonary and the bronchial artery systems is provided. During lung graft retrieval, the bronchoarterial system is not preserved selectively and is inevitably interrupted. Although revascularization of the bronchial artery circulation during transplantation has been shown to be beneficial for airway healing in certain patient populations, ${ }^{3}$ this concept has not been widely accepted in bilateral sequential lung transplantation. Reestablishment of blood flow in the bronchial arteries is technically demanding and has led to inconsistent results. Most centers therefore omit revascularization of the bronchial arteries for lack of feasibility of the procedure. By contrast, semiselective perfusion of the bronchial arteries during organ retrieval would represent a straightforward and promising approach. We hypothesized that a sufficient preservation of the bronchial artery tree might lead to improved bronchial healing and a reduction in the incidence of bronchial anastomotic complications. We therefore developed a novel technique for semiselective bronchial artery perfusion.

Lungs from three consecutive organ donors were accepted for double-lung transplantation and chosen for application of our bronchial artery perfusion technique. After an informative introduction, the participating cardiac and abdominal surgeons had no objections regarding the application of this technique. After standard evaluation by bronchoscopy and macroscopic inspection of the lungs, the aortic arch was dissected, including the proximal supra-aortic branches. The brachiocephalic trunk and left carotid and left subclavian arteries were prepared for clamping. Two separate purse-string sutures were positioned in the distal aortic

\footnotetext{
From the Hannover Thoracic Transplant Program, Division of Thoracicand Cardiovascular Surgery, Hannover Medical School, Hannover, Germany.

Received for publication Dec 19, 2003; accepted for publication June 2, 2004.

Address for reprints: Martin Strueber, MD, Director, Hannover Thoracic Transplant Program, Division of Thoracic- and Cardiovascular Surgery, Hannover Medical School, Carl-Neuberg-Strasse 1, 30623 Hannover, Germany (E-mail: strueber@thg.mh-hannover.de).

J Thorac Cardiovasc Surg 2005;129:456-7

$0022-5223 / \$ 30.00$

Copyright $\odot 2005$ by The American Association for Thoracic Surgery doi:10.1016/j.jtcvs.2004.06.022
}

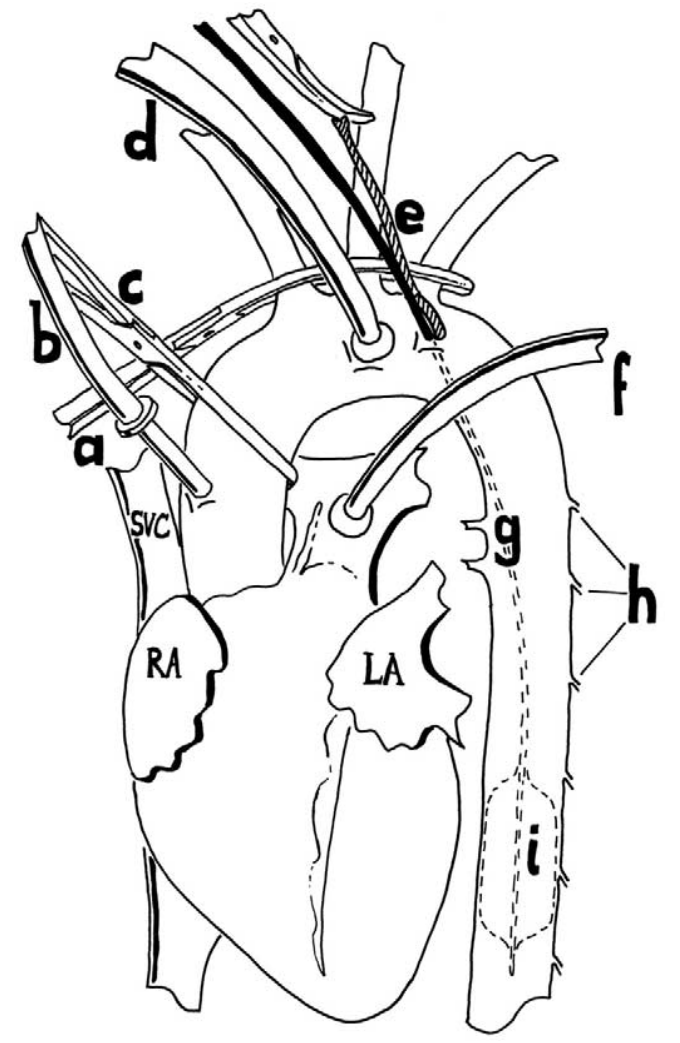

Figure 1. Schematic illustration of intraoperative setting during combined heart and lung harvesting. $d$, Cannula for semiselective bronchial artery perfusion in aortic arch; e, occlusion catheter inside descending aorta in supradiaphragmatic position; $c$, clamp on ascending aorta; $b$, cannula for cardioplegic solution; $a$, clamp on supra-aortic arteries; $f$, cannula in pulmonary artery for antegrade lung perfusion; $S V C$, superior vena cava; $g$, bronchial arteries; $h$, intercostal arteries; $R A$, right atrium; $L A$, left atrium; $i$, occlusion balloon.

arch. An aortic occlusion catheter (8/22F Fogarty occlusion catheter; Edwards Lifesciences Corporation, Irvine, Calif) was introduced and digitally guided into the middle segment of the descending thoracic aorta through both distal purse-string sutures (Figure 1). Consecutively, the standard cannulation procedures for multiorgan retrieval were performed. After opening of the left atrium and the inferior vena cava and crossclamping of the ascending aorta, perfusion with specific organ preservation solutions was initiated. Low-potassium dextran solution $\left(6 \mathrm{~L}\right.$ at $\left.10^{\circ} \mathrm{C}\right)$ was applied for antegrade flush perfusion through the pulmonary artery for 15 minutes. Once perfusion was started, an additional cannula 
was inserted into the proximal purse-string suture in the aortic arch, and the aortic occlusion catheter was inflated to block the descending aorta. The three supra-aortic vessels were crossclamped. Bronchial artery perfusion was simultaneously performed with $2 \mathrm{~L}$ low-potassium dextran solution at $10^{\circ} \mathrm{C}$ through the isolated portion of the aorta. During routine organ harvesting, the bronchial artery perfusion was continued until dissection of the double-lung block off the descending aorta. After completion of the perfusion period, the organs were procured according to standard protocols. In addition to the lungs, in 2 of the 3 cases the heart was retrieved for transplantation, and from all 3 donors multiple abdominal organs were also transplanted.

Bilateral sequential lung transplantation was performed in all 3 cases without the use of cardiopulmonary bypass. Ischemic times were within 5 hours for the right lungs and within 7 hours for the left lungs. The recipients were extubated $19 \pm 4$ hours after transplantation. The early follow-up bronchoscopies on days 0,7 , and 14 after transplantation revealed regular bronchial mucosal findings. Bronchial anastomoses showed normal healing.

This novel approach to semiselective bronchial artery perfusion is a feasible technique that may improve preservation of the graft airways. The exact volume of perfusate reaching the bronchial arteries remains unknown because of the concurrent flows through the intercostal and pulmonary arteries. The procedure does not interfere with standard protocols of thoracic and abdominal organ preservation. The beneficial impact on bronchial healing after lung transplantation remains a matter of speculation at this time and needs to be investigated in future studies. The aim of this report was to introduce this novel approach to lung preservation, which we now routinely apply in our program.

\section{References}

1. Schäfers HJ, Schäfer CM, Zink C, Haverich A, Borst HG. Surgical treatment of airway complications after lung transplantation. $J$ Thorac Cardiovasc Surg. 1994;107:1476-80.

2. Date H, Trulock EP, Arcidi JM, Sundaresan S, Cooper JD, Patterson GA. Improved airway healing after lung transplantation: an analysis of 348 bronchial anastomoses. J Thorac Cardiovasc Surg. 1995;110: 1424-33.

3. Nørgaard MA, Efsen F, Andersen CB, Svendsen UG, Pettersson G. Medium-term patency and anatomic changes after direct bronchial artery revascularization in lung and heart-lung transplantation with the internal thoracic artery conduit. J Thorac Cardiovasc Surg. 1997;114: 326-31. 